\title{
Latest evidence on immunotherapy for cholangiocarcinoma (Review)
}

\author{
XURUI GUO and WEIZHANG SHEN \\ Department of Oncology and Hematology, The Second Hospital of Jilin University, Changchun, Jilin 130041, P.R. China
}

Received July 12, 2020; Accepted October 6, 2020

DOI: $10.3892 / \mathrm{ol} .2020 .12244$

\begin{abstract}
Cholangiocarcinoma (CCA) is a type of aggressive tumor that involves the intrahepatic, perihilar and distal biliary tree, and is usually diagnosed at an advanced stage. The standard first-line systemic therapy for patients with advanced CCA is a combination of gemcitabine and cisplatin; targeted therapies and angiogenesis inhibitors are not widely used clinically at present. However, with the development of precision medicine, immunotherapy has started to play a more important role. Programmed cell death protein 1 inhibitors are now considered a good therapeutic option for CCA. Treatments using chimeric antigen receptor $\mathrm{T}$ cells, bispecific antibodies, oncolytic viruses and cancer vaccines have also achieved satisfactory results. In addition, combinations of immunotherapy with a variety of conventional therapies have shown some efficacy, and several studies have provided insights into their use in antitumor therapy. Although there are numerous challenges in the treatment of advanced CCA, immunotherapy remains a noteworthy breakthrough. The current evidence on the immunotherapy of CCA is discussed in the present review.
\end{abstract}

\section{Contents}

1. Introduction

2. Immunotherapy

3. Combined therapy

4. Conclusions

\section{Introduction}

Cholangiocarcinoma (CCA) is a type of aggressive tumor that involves the intrahepatic, perihilar and distal biliary tree (1).

Correspondence to: Dr Weizhang Shen, Department of Oncology and Hematology, The Second Hospital of Jilin University, 218 Ziqiang, Changchun, Jilin 130041, P.R. China

E-mail: shenweizhang2015@126.com

Key words: cholangiocarcinoma, immunotherapy, programmed cell death protein 1 , chimeric antigen receptor $\mathrm{T}$ cells, bispecific antibody, oncolytic viruses, cancer vaccines
The mass-forming type is the most common growth pattern of intrahepatic CCA (iCCA), which accounts for $~ 65 \%$ of all cases. Periductal infiltrating and intraductal growth are also commonly observed patterns of growth, on the basis of gross appearance (2). Perihilar CCA (pCCA) and distal CCA (dCCA) often appear as flat or unclearly defined nodular sclerosing tumors that diffusely infiltrate adjacent structures (2). Biliary intraepithelial neoplasia and intraductal papillary neoplasm of the bile duct are considered to be premalignant lesions of invasive CCA (3).

CCA accounts for $3 \%$ of all gastrointestinal tumors (4). Globally, hepatobiliary malignancies account for $13 \%$ of cancer-associated deaths, and 10-20\% of these are attributable to CCA (4). A study revealed that in the USA, the highest incidence of CCA is observed in Hispanic and Asian individuals (2.8/100,000 and 3.3/100,000, respectively), with the incidence and mortality rates of men being slightly higher than those of women (5). Cirrhosis, viral hepatitis (including hepatitis B and C), alcohol, tobacco, metabolic factors, non-alcoholic fatty liver disease, diabetes, obesity and genetic susceptibility are thought to be associated with the occurrence of the disease (6). Inflammation and cholestasis are the main mediating factors in the development of CCA (7). A large, US population-based case-control study of $\geq 66$-year-old patients who received Medicare demonstrated that autoimmunity problems, including systemic lupus erythematosus and vitiligo, are also associated with the risk of hepatobiliary cancer (8).

CCA is usually diagnosed at an advanced stage. There are nearly no symptoms at the early stage, and it is also difficult to detect by imaging examination. Patients with CCA often suffer from abdominal discomfort, appetite loss, weight loss and other clinical symptoms, the most typical of which is painless jaundice (9). It is important to accurately determine a treatment plan for the disease in conjunction with imaging examination.

Surgery is usually the preferred treatment option, but liver transplantation following neoadjuvant chemotherapy is also an option for a small number of patients with pCCA (1). The standard first-line systemic therapy for patients with advanced CCA is a combination of gemcitabine and cisplatin; targeted therapies and angiogenesis inhibitors have not been widely used in the clinic so far. It is well known that escape from immune surveillance contributes to the occurrence of tumors and, with the development of precision medicine, immunotherapy serves an important role in tumor treatment (10). The 
tumor microenvironment (TME) delivers survival signals to the tumor and might create a barrier to the delivery of chemotherapy (1). With the help of genetic profiling and classifications, the use of targeted therapies, radiation treatments and/or immunotherapy might help to improve the survival outcomes of patients with this otherwise devastating malignancy (1).

\section{Immunotherapy}

Immune checkpoint inhibitors (ICIs). Programmed cell death protein 1 (PD-1) and T lymphocyte-associated antigen 4 are the most common ICI drug targets. The present review focuses on PD-1 and its details.

The expression of PD-1 by tumor-infiltrating lymphocytes is associated with poor prognosis (11). A study found that tumor cells from patients with occupational CCA exhibited immune-escape behavior via the PD-1/programmed death-ligand 1 (PD-L1) axis (12). Another study confirmed the proliferation of cluster of differentiation (CD) $8^{+} \mathrm{T}$ cells after blockade of the PD-1 pathway (13). This phenomenon suggests that PD-1 inhibitors may promote the monitoring of tumor cells by increasing the recruitment of immune cells, and that immunotherapy with checkpoint molecule-specific monoclonal antibodies (mAbs) can be effective for CCA treatment (14). However, PD-1/PD-L1 inhibitors are not beneficial for the treatment of tumors in every patient (15). Pembrolizumab is a humanized immunoglobulin G4 (IgG4) $\kappa$ anti-PD1 mAb. It does not trigger any cytotoxic activity, since the binding of pembrolizumab to PD-1 does not engage Fc receptors or activate the complement system. Pembrolizumab has now been studied in clinical trials for multiple types of tumor (16). According to a report on the KEYNOTE-158 (NCT02628067) and KEYNOTE-028 (NCT02054806) clinical trials, pembrolizumab has durable antitumor activity in $6-13 \%$ of patients with advanced biliary tract cancer (BTC), regardless of PD-L1 expression; it also has manageable toxicity (17). Nivolumab is a fully human IgG4 anti-PD-1 monoclonal antibody that has been approved for multiple advanced malignancies, including melanoma, non-small cell lung cancer, renal cell cancer, Hodgkin's lymphoma, squamous head and neck cancer and urothelial carcinoma (18). In a phase II clinical trial (NCT02829918) of nivolumab in patients with advanced refractory BTC, the objective response rate (ORR) of monotherapy with nivolumab was $11 \%$, including a single case of unconfirmed partial response (PR), with a disease control rate (DCR) of $50 \%$, as determined by central independent review. That study also reported that a median progression-free survival (mPFS) of 3.68 months and a median overall survival (OS) among the intention-to-treat population of 14.24 months (19).

Other biomarkers also require consideration. Indicators of the effectiveness of ICIs in patients with advanced CCA include the expression of PD-L1, DNA mismatch repair (MMR) defects and/or high microsatellite instability (MSI-H) (20). A phase II study showed that the clinical benefit of pembrolizumab can be predicted by MMR status (21). Tumor mutational burden (TMB) is also a common evaluation indicator of whether immunotherapy should be administered. The genomes of cancers with MMR deficiency contain exceptionally high numbers of somatic mutations (22). Certain studies have shown that the total number of somatic mutations (mutational load) is associated with the clinical response to ICI therapies in several types of tumors $(23,24)$. However, another study showed that, as compared with MSI or even the TMB, the presence of immunogenic neoepitopes may be a more persuasive biomarker for immunotherapeutic response (25). In addition, tumor type may no longer be a major factor for determining the response to ICI (22).

Chimeric antigen receptor $(C A R) T$ cells. The emergence of CAR T cells has revitalized immunotherapy. Despite the toxicity profile and huge expense of CAR T cells, the clinical efficacy of CAR T cells in the treatment of refractory hematological malignancies is satisfactory. In the past few years, it has been discovered that improved second-generation CARs, which usually comprise an antibody-derived single-chain variable fragment linked with intracellular signaling molecules, are able to activate T-cell effector functions (26). CD28- and 4-1BB-derived domains are the most commonly used costimulatory domains. 4-1BB-containing CAR T cells are thought to have superior persistence, but the protein phosphorylation changes of signaling intermediates induced by CD28-CAR T cells are stronger and faster (26). Third-generation CAR T cells containing both CD28 and 4-1BB costimulatory domains have been demonstrated to be safe and effective at every dose level tested (27). Although fourth generation CARs, also known as $\mathrm{T}$ cells redirected for universal cytokine-mediated killing, and fifth generation CARs have gradually been developed, it is unclear whether they have the same clinical effects as previous generations in malignant diseases (28). Fifth generation CARs are based on the second generation of CARs, but they contain a truncated cytoplasmic IL-2 receptor $\beta$-chain domain with a binding site for the transcription factor STAT3; the antigen-specific activation of this receptor effectively provides three synergistic signals (TCR, co-stimulatory and cytokine signals) required to drive full T-cell activation and proliferation (28). Researchers have been exploring whether CAR T cells have the same anticancer effect in solid tumors as in blood malignancies, while facing several challenges (28). The same or different types of cancer may have different TMEs. Enhancing the infiltration of tumors by $\mathrm{T}$ cells has been suggested as a potential strategy for increasing the efficacy of tumor immunotherapy (29). Clinical trials of specific CAR $T$ cell treatments for a number of solid tumor types have been conducted $(30,31)$. The use of CAR T-cell cocktail immunotherapy may be a feasible option for patients with CCA, but toxicity requires further investigation (NCT01869166 and NCT02541370) (32). A phase I study of CAR-epidermal growth factor receptor (EGFR) T-cell therapy for the treatment of patients with EGFR-positive advanced unresectable, relapsed/metastatic BTC showed that 1/17 evaluable patients achieved complete remission (CR) and 10 achieved stable disease (SD). The mPFS was 4 months from the first cycle of treatment. This result demonstrates that this treatment is effective for CCA (33). At present, although there is substantial evidence from clinical trials showing the potential of CAR T-cell treatment, the evidence to support its use for the treatment of advanced CCA is not sufficient for clinical 
practice; therefore, more clinical trials are required to ensure the safe application of CAR T-cell therapy.

Bispecific antibodies. Bispecific antibodies that are able to recognize two different epitopes have a broad range of applications. Great progress has been made in the treatment of hematological malignancies and solid tumors with T cell-redirecting bispecific antibodies (TRBAs) (34). Different TRBA formats have the same essential aim; directing CD3 T cells to target tumor cells (34). A recent study on prostate tumor-specific antigen demonstrated that the combination of CD3 bispecific antibodies and anti-4-1BB co-stimulation is a successful therapeutic approach for the treatment of solid tumors (35). Novel IgG-based $1+1$ MG1122-A and $2+1$ MG1122-B bispecific antibodies against mesothelin and $\mathrm{CD} 3 \varepsilon$ have been designed, and this type of construction has been shown to improve antitumor efficacy and tumor targeting; it may also reduce systemic toxicity (36). A study by Thakur et al (37) verified that anti-human epidermal growth factor receptor 2 (HER2) bispecific antibody- or anti-EGFR bispecific antibody-armed CD19-CAR T cells (CART 19) aimed at multiple $\mathrm{HER}^{+} / \mathrm{EGFR}^{+} / \mathrm{CD}^{-} 9^{-}$tumor targets had specific cytotoxicity, and that the CART 19 model improved the survival of the $\mathrm{T}$ cells, which were even resistant to exhaustion under certain conditions. The results of several other studies have also indicated that bispecific antibodies provide satisfactory effects and have a wide range of applications $(38,39)$. In 2003 , a study reported that an anti-mucin 1/anti-CD3 bispecific antibody indirectly increased the antitumor activity of a 4-1BB ligand-encoding adenovirus in CCA (40). However, no other findings about bispecific antibodies in CCA have yet been reported. We hypothesize that in the future, greater success will be achieved with bispecific antibodies in the treatment of CCA.

Oncolytic viruses $(O V s)$ and cancer vaccines. OV therapy has emerged due to the disadvantages of traditional chemotherapy. This type of treatment uses viruses with a natural or engineered ability to infect and kill cancer cells (41). Greater knowledge of the function of viral genes and advancements in molecular biology have significantly improved the safety and efficacy of OVs. The basis of the therapeutic efficacy of OVs is considered to be the recruitment of T cells and induction of tumor-reactive immunity (42). Studies have indicated that delivering OVs into the tumor can facilitate a strong and durable response against the tumor by stimulating the activity of the immune system $(43,44)$. Talimogene laherparepvec is the only approved OV in the US to date (45). In addition, the use of an $\mathrm{OV}$ in combination with inhibition of transforming growth factor- $\beta$ signaling is thought to increase the efficacy of immunotherapy (42). According to a recent study, a novel Vaccinia virus known as $V V \Delta T K \Delta N 1 L$, with thymidine kinase and N1L gene deletions and armed with interleukin 12 , successfully prevented the recurrence of tumors in surgical models of head and neck cancer in Syrian hamsters (46). This study laid an important experimental foundation for the development of new drugs in the treatment of solid tumors. Although good progress has been made with this antitumor method, a number of areas that require improvement and further study remain. Reinforcing the selective replication strength of OVs, constructing efficient OV delivery platforms, maintaining the balance between antiviral and antitumor immunity, overcoming the immunosuppressive TME and enhancing oncolysis are some directions of research for the continuous improvement of OV therapy (47). For example, although systemic injection has been proposed to be the best route of administration for an $\mathrm{OV}$, local (intratumoral) injection is the most used route of administration (48). Preclinical studies investigating the oncolytic effect of a measles vaccine virus and three survivin-based conditionally replicative adenoviruses in CCA reported the possibility of treatment with OV $(49,50)$. More trials are required to verify the safety and efficacy of these agents.

Vaccines for cancer are generally developed with a focus on clearing active disease rather than preventing disease (51). There is some evidence that dendritic cell (DC) vaccines are efficacious in tumor therapy; for example, Sipuleucel-T has been shown to prolong the median OS of patients with prostate cancer (51). A non-randomized uncontrolled study of another DC-based immunotherapy in patients with BTC showed that the treatment was safe; however, 0/65 patients showed a CR at 3 months after the first vaccination. The PR and SD rates were 6 and 23\% respectively, and the DCR and ORR were 29 and $6 \%$, respectively (52). In addition, honokiol may be able to enhance the therapeutic effect of DC-based vaccines (53). In 2012, a study demonstrated that a DC vaccine in combination with activated T-cell transfer could be feasible and effective for preventing recurrence and achieving long-term survival in patients with iCCA following surgery (54). These studies provide evidence to indicate that tumor vaccines can be helpful for patients with CCA. Clinical trials of immunotherapy in CCA are shown in Table I.

\section{Combined therapy}

With the continuous development of biomedical technology and the discovery of some deficiencies in clinical practice, combination therapy involving immunotherapy has the potential to provide improved clinical effects in numerous conditions.

Immunotherapy plus chemotherapy. For patients with advanced-stage CCA that is not suitable for treatment with surgical or locoregional interventions, gemcitabine combined with cisplatin or oxaliplatin is the standard therapy; however, the response to this chemotherapy regimen is unsatisfactory. There is evidence that chemotherapy combined with immunotherapy may result in a good therapeutic response in CCA with a high TMB (55). A phase II trial (NCT03311789) studied the clinical response to nivolumab in combination with gemcitabine and cisplatin in 27 response-evaluable patients with BTC, and found an ORR of 55.6\%, including $5 \mathrm{CRs}$ and $10 \mathrm{PRs}$. Of the 6 patients who were resistant to gemcitabine-based or cisplatin-based chemotherapy, one CR and one PR were achieved. This result indicates that, as a PD-1 inhibitor, nivolumab is able to resensitize BTC to gemcitabine and cisplatin chemotherapy (56). No significant survival benefit was observed for this combination when compared with other combinations in clinical trials (56-58). However, it has promising efficacy and a manageable safety profile in 


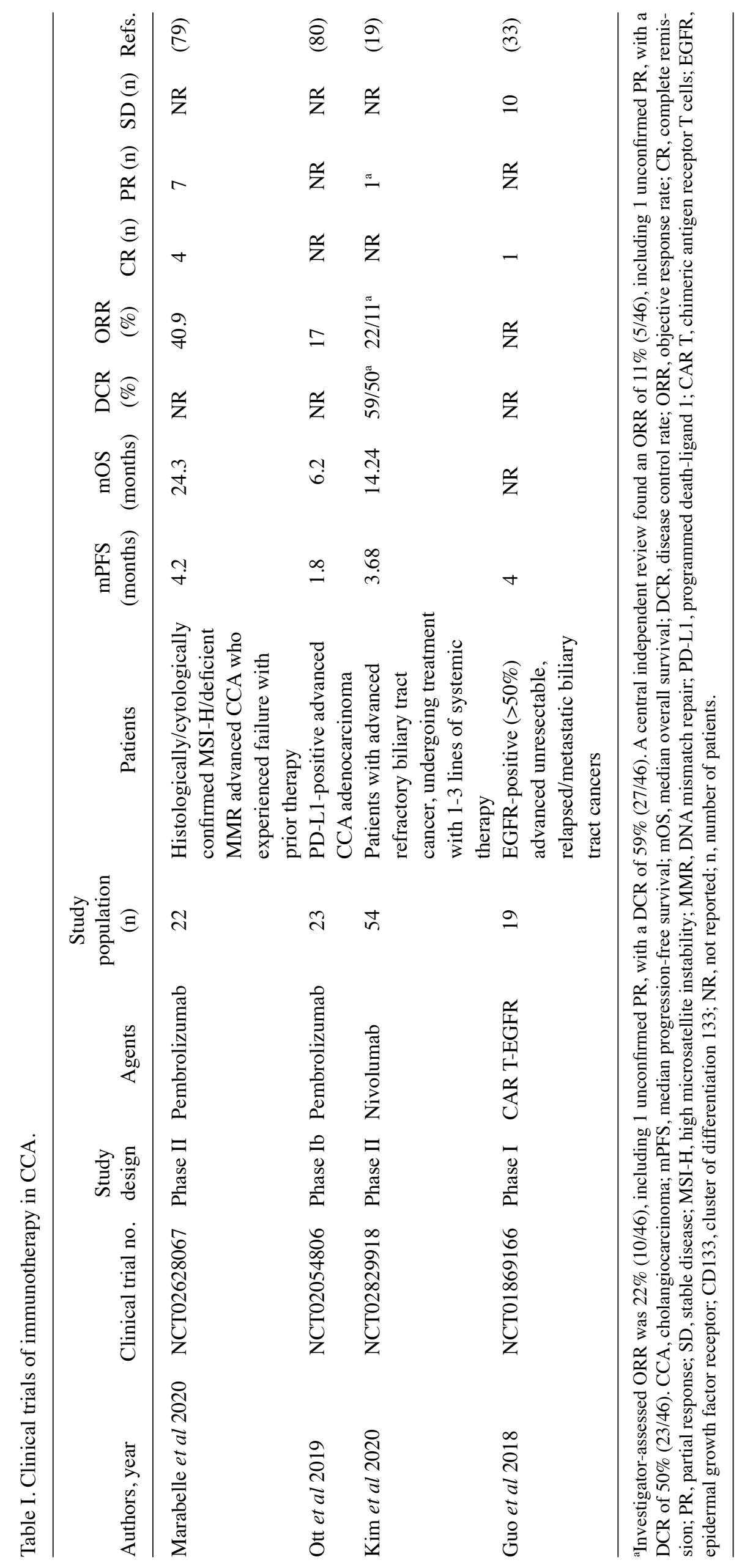


patients with advanced CCA (56). Patients with iCCA with high insertion-deletion ratios can also benefit from treatment with this combination (59).

The aforementioned results may be due to the synergistic effect of chemotherapy and ICIs; it is suggested that the use of an ICI increased the efficacy of gemcitabine, which in turn increased the antigenicity of tumor cells and partially reduced the immunosuppressive effect of chemotherapy, which may be associated with the role of tumor-associated macrophages (56). Therefore, the addition of a PD-1 inhibitor to a chemotherapy regimen can better control the disease, benefit the patients and attenuate resistance to chemotherapy. However, as discussed above, the adverse reactions of ICIs are also a challenge that must be considered. Therefore, comprehensive management should be conducted to maximize disease benefits and minimize adverse reactions in clinical practice.

The combination of chemotherapy and DCs is another feasible combined therapy. In one clinical study of patients with BTCs, the median survival time (MST) was prolonged by DC-based immunotherapy and chemotherapy, with a MST from the first vaccination of 8.2 months with chemotherapy compared with 5.3 months without chemotherapy. In $50(77 \%)$ of the 65 patients treated with chemotherapy and DC vaccine, the DCR and ORR were 34 and $8 \%$, respectively. In the remaining 15 patients treated with the DC vaccine but without chemotherapy, the DCR and ORR were 13 and $0 \%$, respectively (52). In this study, a comprehensive analysis of associated influencing factors, including albumin level, C-reactive protein level, fever after inoculation and nutritional index, was performed. The clinical efficacy of the DC vaccine following combined chemotherapy was improved compared with that of the DC vaccine alone, but this treatment did not have a clear advantage in curative effect, as compared with chemotherapy regimens combined with ICI.

Immunotherapy plus radiotherapy. Radiotherapy is a common antitumor treatment. When radiotherapy is administered, the sensitivity of the immune system to the tumors is increased (60). As mentioned above, for patients with a high TMB, MSI-H, deficient MMR and/or positive PD-L1 expression, treatment with a PD-1 inhibitor may provide satisfactory results. Notably and unexpectedly, a case report showed that radiotherapy can improve the efficacy of immunotherapy in patients with late-stage or recurrent iCCA with low TMB, proficient MMR, microsatellite stability and negative PD-L1 expression status (61). This result expands the scope of treatment for CCA.

Jarnagin et al (62) demonstrated that immunotherapy and radiotherapy have synergistic effects in the treatment of CCA. In their study, which was reported in 2006, the clinical application of oncolytic herpes simplex virus combined with external beam radiation therapy yielded satisfactory clinical results. This efficacy was hypothesized to be associated with the radiation-induced upregulation of growth arrest and DNA damage protein 34 , which promoted tumoricidal activity in viral strains deficient in the $\gamma(1) 34.5$ gene (62). Since then, this type of treatment combination has been rarely used. However, according to the results obtained to date, immunotherapy combined with radiotherapy has a certain efficacy and can continue to be considered as a potential direction for malignant tumor treatment.
Immunotherapy plus small molecule inhibitors. For CCA, genes affected by congenital mutations/polymorphisms include ATP binding cassette subfamily B member 4, ATP binding cassette subfamily B member 11, ATP binding cassette subfamily $\mathrm{C}$ member 2, ATPase phospholipid transporting 8B1, cyclooxygenase 2, cytochrome P450 1A2 and glutathione S-transferase $\omega-1$, and those affected by acquired mutations include adenomatous polyposis coli, AT-rich interaction domain 1A, axin 1, BRCA1 associated protein 1, BCL-2, BCL2 like 1, BRAF, EGFR (ERBB1), fibroblast growth factor receptor 2 (FGFR2), isocitrate dehydrogenase 1 (IDH1), IDH2 and tumor protein 53 (7). IDH1/2 mutations and FGFR2 fusions are features typically observed in small-duct iCCA, while they are usually absent in extrahepatic CCA. Furthermore, the mutation frequency of KRAS seems to be higher in pCCA and dCCA than in iCCA (2). Molecular therapy targeting these specific genes has demonstrated certain therapeutic effects (63), and immune drugs have also shown a promising clinical efficacy against these genes. A clinical trial (NCT01752920) was conducted in adult patients with unresectable iCCA and FGFR2 fusion to investigate the preliminary therapeutic activity of derazantinib, an orally bioavailable, multi-kinase inhibitor with potent pan-FGFR activity. The trial reported an ORR of $20.7 \%$, DCR of $82.8 \%$ and estimated mPFS of 5.7 months (64). Abou-Alfa et al (65) reported the result of a clinical trial (NCT02924376), in which pemigatinib, a selective, potent oral inhibitor of fibroblast growth factor receptor (FGFR)1, 2 and 3, exhibited good therapeutic potential for CCA. Lenvatinib is a small-molecule tyrosine kinase inhibitor that inhibits VEGFR1-3, FGFR1-4, platelet-derived growth factor receptor $\alpha$, stem cell factor receptor and RET (66). In a patient with recurrent iCCA with bone metastasis, amelioration of the disease was observed following treatment with nivolumab immunotherapy plus lenvatinib (67). This suggests that immunotherapy plus molecular-targeted therapy could be an option for the late-line treatment of CCA. However, there is as yet no relevant evidence to support the combination of molecular targeted therapy and immunotherapy for the treatment of CCA; nor is there any clear evidence supporting its safety, efficacy and the benefits to patients. Despite this, it is potentially a feasible approach, and is worthy of further research.

Combinations of multiple immunotherapy drugs. In recent years, research into the efficacy of combinations of multiple immunotherapy drugs has begun, and their respective strengths are emerging. A clinical trial, NCT02923934, explored the efficacy and safety of a combination of two immunotherapy drugs, nivolumab and ipilimumab, for the treatment of CCA. This phase II clinical trial reported an ORR of $23 \%$, DCR of $44 \%$, mPFS of 2.9 months and OS of 5.7 months. Notably, all patients who responded had received prior chemotherapy, and none of them had a microsatellite unstable tumor (68).

Although the combination of immunotherapy drugs proved clinically effective in the study, dual-drug immunization was not superior to single-drug immunotherapy, with the exception of its effects on ORR. Dual-drug immunotherapy remains a distant prospect for patients with CCA.

Immunotherapy plus antiangiogenic (AA) therapy. AA therapy is an type of antitumor treatment that is effective against most 


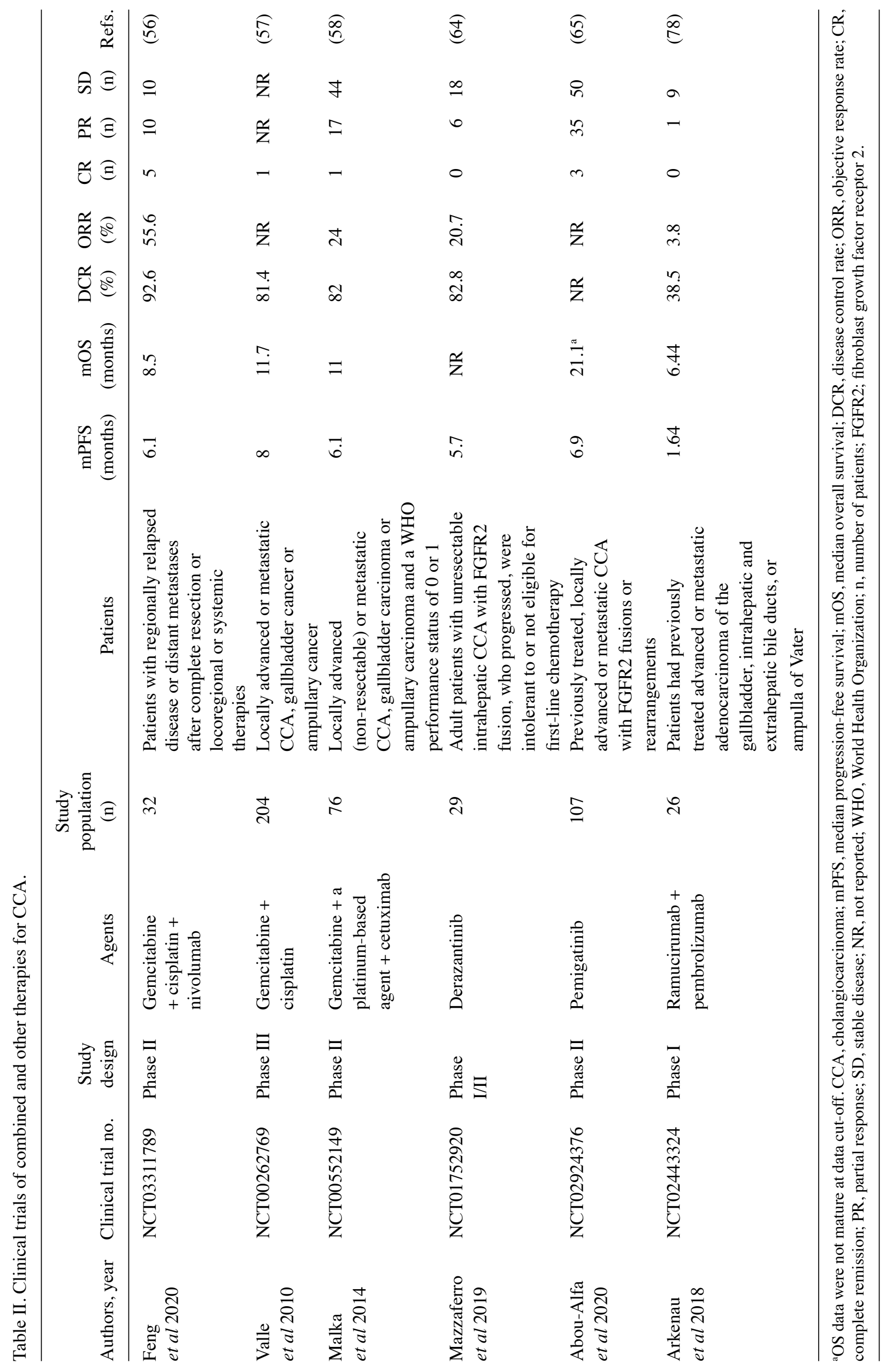


tumors. The growth of cancer cells depends on blood vessels to supply and transport nutrients, and microvessel density is associated with cancer progression, metastasis and prognosis (69). The discovery that vascular endothelial growth factor (VEGF) is an important mediator of angiogenesis prompted research into the therapeutic targeting of this pathway; furthermore, the expression of VEGF may be involved in hematogenic metastasis in CCA $(70,71)$. VEGF has been demonstrated to be significantly associated with intrahepatic metastasis in iCCA, while in pCCA, VEGF-A expression is thought to be upregulated and associated with increased vascular density. This suggests that AA treatments may achieve a good response in pCCA (69). A previous study has revealed that IDH1/2 mutations are associated with iCCA, KRAS mutations are associated with pCCA, and protein kinase CAMP-activated catalytic subunit $\alpha$ or $\beta$ gene fusions preferentially occur in eCCA (69). Hypoxia is the main stimulus for the production of VEGF, which leads to expression of the transcription factor hypoxia inducible factor 1- $\alpha$ (72). In addition to the VEGF, MEK/ERK, Yes-associated protein/Hippo and other pathways are also involved in angiogenesis (69), and may be potential targets for AA therapy. Bevacizumab, ramucirumab, sorafenib, regorafenib and several other new drugs are being used or studied for AA therapy, some of which have a good clinical efficacy (73-75).

Studies have shown that PD-1/PD-L1 inhibitors may enhance the antitumor effects of AA drugs (76,77). A preclinical study demonstrated that AA therapy also can improve anti-PD-L1 treatment in certain conditions by supporting vascular changes, such as vessel normalization and high endothelial venule formation, that facilitate enhanced cytotoxic T-cell infiltration, activity and tumor cell destruction (77). The simultaneous blockade of VEGF receptor-2 and either PD-1 or PD-L1 has been shown to induce additive antitumor effects (78). A non-randomized, open-label, phase I trial of ramucirumab and pembrolizumab was the first to combine AA therapy with an ICI to treat advanced BTC. The ORR was $3.8 \%$, with mPFS and OS times of 1.64 and 6.44 months, respectively (78). In addition, the occurrence of adverse events was not negligible. These results suggest that although this type of combination treatment has a certain efficacy, it does not have a significant advantage over the aforementioned treatments, and may not be an optimal choice for advanced treatment. However, it remains to be seen whether combinations of immunotherapy agents with other AA drugs against other targets will achieve different results. Clinical trials for combined therapy in CCA are shown in Table II.

\section{Conclusions}

Although numerous challenges remain in the treatment of advanced CCA, immunotherapy is a noteworthy breakthrough. As discussed in this review, immunotherapy with a single drug, multiple drugs or a combination of traditional therapies can sometimes be an option for the treatment of CCA. With further theoretical research and evidence to validate their efficacy and safety, new treatment combinations may become the basis and direction for future studies. Combining more treatments may provide further benefits to patients with CCA. These new treatment combinations may also have implications for other types of cancer.

\section{Acknowledgements}

Not applicable.

\section{Funding}

No funding was received.

\section{Availability of data and materials}

Not applicable.

\section{Authors' contributions}

XG performed formal analysis, searched the literature and wrote the original draft. WS was responsible for conceptualization and literature search, as well as reviewing and editing the manuscript. Both authors read and approved the final manuscript.

\section{Ethics approval and consent to participate}

Not applicable.

\section{Patient consent for publication}

Not applicable.

\section{Competing interests}

The authors declare that they have no competing interests.

\section{References}

1. Rizvi S, Khan SA, Hallemeier CL, Kelley RK and Gores GJ: Cholangiocarcinoma-evolving concepts and therapeutic strategies. Nat Rev Clin Oncol 15: 95-111, 2018.

2. Kendall T, Verheij J, Gaudio E, Evert M, Guido M, Goeppert B and Carpino G: Anatomical, histomorphological and molecular classification of cholangiocarcinoma. Liver Int 1: 7-18, 2019.

3. Nakanishi Y, Zen Y, Kondo S, Itoh T, Itatsu K and Nakanuma Y: Expression of cell cycle-related molecules in biliary premalignant lesions: Biliary intraepithelial neoplasia and biliary intraductal papillary neoplasm. Hum Pathol 39: 1153-1161, 2008.

4. Rizvi S and Gores GJ: Pathogenesis, diagnosis, and management of cholangiocarcinoma. Gastroenterology 145: 1215-1229, 2013.

5. Everhart JE and Ruhl CE: Burden of digestive diseases in the United States part III: Liver, biliary tract, and pancreas. Gastroenterology 136: 1134-1144, 2009.

6. Massarweh NN and El-Serag HB: Epidemiology of hepatocellular carcinoma and intrahepatic cholangiocarcinoma. Cancer Control 24: 1073274817729245, 2017.

7. Labib PL, Goodchild G and Pereira SP: Molecular Pathogenesis of Cholangiocarcinoma. BMC Cancer 19: 185, 2019.

8. McGee EE, Castro FA, Engels EA, Freedman ND, Pfeiffer RM, Nogueira L, Stolzenberg-Solomon R, McGlynn KA, Hemminki K and Koshiol J: Associations between autoimmune conditions and hepatobiliary cancer risk among elderly US adults. Int J Cancer 144: 707-717, 2019.

9. Brandi G, Venturi M, Pantaleo MA and Ercolani G; GICO: Cholangiocarcinoma: Current opinion on clinical practice diagnostic and therapeutic algorithms: A review of the literature and a long-standing experience of a referral center. Dig Liver Dis 48: 231-241, 2016.

10. Saeed A, Park R, Al-Jumayli M, Al-Rajabi R and Sun W: Biologics, immunotherapy, and future directions in the treatment of advanced cholangiocarcinoma. Clin Colorectal Cancer 18: 81-90, 2019. 
11. Ohaegbulam KC, Assal A, Lazar-Molnar E, Yao Y and Zang X Human cancer immunotherapy with antibodies to the PD-1 and PD-L1 pathway. Trends Mol Med 21: 24-33, 2015.

12. Sato Y, Kinoshita M, Takemura S, Tanaka S, Hamano G, Nakamori S, Fujikawa M, Sugawara Y, Yamamoto T, Arimoto A, et al: The PD-1/PD-L1 axis may be aberrantly activated in occupational cholangiocarcinoma. Pathol Int 67: $163-170,2017$

13. Im SJ, Hashimoto M, Gerner MY, Lee J, Kissick HT, Burger MC, Shan Q, Hale JS, Lee J, Nasti TH, et al: Defining CD8 ${ }^{+}$T cells that provide the proliferative burst after PD-1 therapy. Nature 537: 417-421, 2016.

14. Sabbatino F, Villani V, Yearley JH, Deshpande V, Cai L, Konstantinidis IT, Moon C, Nota S, Wang Y, Al-Sukaini A, et al: PD-L1 and HLA class I antigen expression and clinical course of the disease in intrahepatic cholangiocarcinoma. Clin Cancer Res 22: 470-478, 2016.

15. Yi M, Jiao D, Xu H, Liu Q, Zhao W, Han X and Wu K: Biomarkers for predicting efficacy of PD-1/PD-L1 inhibitors. Mol Cancer 23: 129, 2018.

16. Kwok G, Yau TC, Chiu JW, Tse E and Kwong YL: Pembrolizumab (Keytruda). Hum Vaccin Immunother 12: 2777-2789, 2016.

17. Piha-Paul SA, Oh DY, Ueno M, Malka D, Chung HC, Nagrial A Kelley RK, Ros W, Italiano A, Nakagawa K, et al: Efficacy and safety of pembrolizumab for the treatment of advanced biliary cancer: Results from the KEYNOTE-158 and KEYNOTE-028 studies. Int J Cancer 15: 2190-2198, 2020.

18. Finkelmeier F, Waidmann O and Trojan J: Nivolumab for the treatment of hepatocellular carcinoma. Expert Rev Anticancer Ther 18: 1169-1175, 2018.

19. Kim RD, Chung V, Alese OB, El-Rayes BF, Li D, Al-Toubah TE, Schell MJ, Zhou JM, Mahipal A, Kim BH, et al: A phase 2 multi-institutional study of nivolumab for patients with advanced refractory biliary tract cance. JAMA Oncol 6: 1-8, 2020.

20. Pellino A, Loupakis F, Cadamuro M, Dadduzio V, Fassan M Guido M, Cillo U, Indraccolo S and Fabris L: Precision medicine in cholangiocarcinoma. Transl Gastroenterol Hepatol 3: 40, 2018.

21. Le DT, Uram JN, Wang H, Bartlett BR, Kemberling H, Eyring AD, Skora AD, Luber BS, Azad NS, Laheru D, et al: PD-1 blockade in tumors with mismatch-repair deficiency. N Engl J Med 372: 2509-2520, 2015.

22. Le DT, Durham JN, Smith KN, Wang H, Bartlett BR, Aulakh LK, Lu S, Kemberling H, Wilt C, Luber BS, et al: Mismatch repair deficiency predicts response of solid tumors to PD-1 blockade. Science 357: 409-413, 2017.

23. Snyder A, Makarov V, Merghoub T, Yuan J, Zaretsky JM, Desrichard A, Walsh LA, Postow MA, Wong P, Ho TS, et al: Genetic basis for clinical response to CTLA-4 blockade in melanoma. N Engl J Med 371: 2189-2199, 2014.

24. Zhu B, Tang L, Chen S, Yin C, Peng S, Li X, Liu T, Liu W, Han C, Stawski L, et al: Targeting the upstream transcriptional activator of PD-L1 as an alternative strategy in melanoma therapy. Oncogene 37: 4941-4954, 2018.

25. Dudley JC, Lin MT, Le DT and Eshleman JR: Microsatellite instability as a biomarker for PD-1 blockade. Clin Cancer Res 22: 813-820, 2016

26. Stoiber S, Cadilha BL, Benmebarek MR, Lesch S, Endres S and Kobold S: Limitations in the design of chimeric antigen receptors for cancer therapy. Cells 8: 472, 2019.

27. Gomes da Silva D, Mukherjee M, Madhuwanti S, Dakhova O, Liu H, Grilley B, Gee AP, Neelapu SS, Rooney CM, Heslop HE, et al: Direct comparison of in vivo fate of second and third-generation CD19-specific chimeric antigen receptor (CAR)-T cells in patients with B-cell non-hodgkin lymphoma (B-NHL): Reversal of toxicity from tonic signaling. Biol Blood Marrow Transplant 23: S55-S56, 2017.

28. Tokarew N, Ogonek J, Endres S, von Bergwelt-Baildon M and Kobold S: Teaching an old dog new tricks: Next-generation CAR T cells. Br J Cancer 120: 26-37, 2019.

29. Zhang J, Endres S and Kobold S: Enhancing tumor T cell infiltration to enable cancer immunotherapy. Immunotherapy 11: 201-213, 2019.

30. Bielamowicz K, Fousek K, Byrd TT, Samaha H, Mukherjee M, Aware N, Wu MF, Orange JS, Sumazin P, Man TK, et al: Trivalent CAR T cells overcome interpatient antigenic variability in glioblastoma. Neuro Oncol 20: 506-518, 2018.

31. Yu S, Li A, Liu Q, Li T, Yuan X, Han X and Wu K: Chimeric antigen receptor $\mathrm{T}$ cells: A novel therapy for solid tumors. J Hematol Oncol 10: 78, 2017.
32. Feng KC, Guo YL, Liu Y, Dai HR, Wang Y, Lv HY, Huang JH, Yang QM and Hanet WD: Cocktail treatment with EGFR-specific and CD133-specific chimeric antigen receptor-modified T cells in a patient with advanced cholangiocarcinoma. J Hematol Oncol 10: 4, 2017.

33. Guo Y, Feng K, Liu Y, Wu Z, Dai H, Yang Q, Wang Y, Jia H and Han W: Phase I study of chimeric antigen receptor-modified $\mathrm{T}$ cells in patients with EGFR-positive advanced biliary tract cancers. Clin Cancer Res 24: 1277-1286, 2018.

34. Yu L and Wang J: T cell-redirecting bispecific antibodies in cancer immunotherapy: Recent advances. J Cancer Res Clin Oncol 145: 941-956, 2019.

35. Chiu D, Tavaré R, Haber L, Aina OH, Vazzana K, Ram P, Danton M, Finney J, Jalal S, Krueger P, et al: A PSMA-Targeting CD3 bispecific antibody induces antitumor responses that are enhanced by $4-1 \mathrm{BB}$ costimulation. Cancer Immunol Res 8 : 596-608, 2020 .

36. Yoon A, Lee S, Lee S, Lim S, Park YY, Song E, Kim DS, Kim K and Lim Y: A novel T cell-engaging bispecific antibody for treating mesothelin-positive solid tumors. Biomolecules 10: 399, 2020.

37. Thakur A, Scholler J, Schalk DL, June CH and Lum LG: Enhanced cytotoxicity against solid tumors by bispecific antibody-armed CD19 CAR T cells: A proof-of-concept study. J Cancer Res Clin Oncol 146: 2007-2016, 2020.

38. Lum LG, Thakur A, Elhakiem A, Alameer L, Dinning E and Huang M: Anti-CS1 $x$ anti-CD3 bispecific antibody (BiAb)-armed anti-CD3 activated T cells (CS1-BATs) kill $\mathrm{CSI}^{+}$ myeloma cells and release type-1 cytokines. Front Oncol 10: 544 , 2020.

39. Cebada J,Flores A, Bandala C,Lizaliturri-Flores I, Villa-Ruano N and Perez-Santos M: Bispecific anti-PD-1/LAG-3 antibodies for treatment of advanced or metastatic solid tumors: A patent evaluation of US2018326054. Expert Opin Ther Pat 30: 1-8, 2020.

40. Yoshida H, Katayose Y, Unno M, Suzuki M, Kodama H, Takemura Si, Asano R, Hayashi H, Yamamoto K, Matsuno S, et al: A novel adenovirus expressing human 4-1BB ligand enhances antitumor immunity. Cancer Immunol Immunother 52: 97-106, 2003.

41. Hill C and Carlisle R: Achieving systemic delivery of oncolytic viruses. Expert Opin Drug Deliv 16: 607-620, 2019.

42. Groeneveldt C, van Hall T, van der Burg SH, Ten Dijke P and van Montfoort N: Immunotherapeutic potential of TGF- $\beta$ inhibition and oncolytic viruses. Trends Immunol 41: 406-420, 2020.

43. Marelli G, Howells A, Lemoine NR and Wang Y: Oncolytic viral therapy and the immune system: A double-edged sword against cancer. Front Immunol 9: 866, 2018

44. Cervera-Carrascon V, Quixabeira DC, Havunen R, Santos JM, Kutvonen E, Clubb JH, Siurala M, Heiniö C, Zafar S, Koivula T, et al: Comparison of clinically relevant oncolytic virus platforms for enhancing $\mathrm{T}$ cell therapy of solid tumors. Mol Ther Oncolytics 17: 47-60, 2020.

45. Hamid O, Ismail R and Puzanov I: Intratumoral immunotherapy-update 2019. Oncologist 25: e423-e438, 2020.

46. Ahmed J, Chard LS, Yuan M, Wang J, Howells A, Li Y, Li H, Zhang Z, Lu S, Gao D, et al: A new oncolytic vacciniavirus augments antitumor immune responses to prevent tumor recurrence and metastasis after surgery. J Immunother Cancer 8: $\mathrm{e} 000415,2020$.

47. Zheng M, Huang J, Tong A and Yang H: Oncolytic viruses for cancer therapy: Barriers and recent advances. Mol Ther Oncolytics 15: 234-247, 2019.

48. Reale A, Vitiello A, Conciatori V, Parolin C, Calistri A and Palù G: Perspectives on immunotherapy via oncolytic viruses. Infect Agent Cancer 14: 5, 2019.

49. Lange S, Lampe J, Bossow S, Zimmermann M, Neubert W, Bitzer M and Laueret UM: A novel armed oncolytic measles vaccine virus for the treatment of cholangiocarcinoma. Hum Gene Ther 24: 554-564, 2013

50. Zhu ZB, Chen Y, Makhija SK, Lu B, Wang M, Rivera AA, Yamamoto M, Wang S, Siegal GP, Curiel DT and McDonald JM: Survivin promoter-based conditionally replicative adenoviruses target cholangiocarcinoma. Int J Oncol 29: 1319-1329, 2006.

51. Thomas S and Prendergast GC: Cancer vaccines: A brief overview. Methods Mol Biol 1403: 755-761, 2016.

52. Kobayashi M, Sakabe T, Abe H, Tanii M, Takahashi H, Chiba A, Yanagida E, Shibamoto Y, Ogasawara M, Tsujitani Si, et al: Dendritic cell-based immunotherapy targeting synthesized peptides for advanced biliary tract cancer. J Gastrointest Surg 17: 1609-1617, 2013 
53. Jiraviriyakul A, Songjang W, Kaewthet P, Tanawatkitichai P, Bayan P and Pongcharoen S: Honokiol-enhanced cytotoxic T lymphocyte activity against cholangiocarcinoma cells mediated by dendritic cells pulsed with damage-associated molecular patterns. World J Gastroenterol 25: 3941-3955, 2019.

54. Shimizu K, Kotera Y, Aruga A, Takeshita N, Takasaki K and Yamamoto M: Clinical utilization of postoperative dendritic cell vaccine plus activated T-cell transfer in patients with intrahepatic cholangiocarcinoma. J Hepatobiliary Pancreat Sci 19: 171-178, 2012.

55. Mou H, Yu L, Liao Q, Hou X, Wu Y, Cui Q, Yan N, Ma R, Wang L, Yao $M$ and Wang K: Successful response to the combination of immunotherapy and chemotherapy in cholangiocarcinoma with high tumour mutational burden and PD-L1 expression: A case report. BMC Cancer 18: 1105, 2018.

56. Feng K, Liu Y, Zhao Y, Yang Q, Dong L, Liu J, Li X, Zhao Z, Mei Q and Han W: Efficacy and biomarker analysis of nivolumab plus gemcitabine and cisplatin in patients with unresectable or metastatic biliary tract cancers: Results from a phase II study. J Immunother Cancer 8: e000367, 2020.

57. Valle J, Wasan H, Palmer DH, Cunningham D, Anthoney A Maraveyas A, Madhusudan S, Iveson T, Hughes S, Pereira SP, et al: Cisplatin plus gemcitabine versus gemcitabine for biliary tract cancer. N Engl J Med 362: 1273-1281, 2010.

58. Malka D, Cervera $P$, Foulon $S$, Trarbach $T$, de la Fouchardière $C$, Boucher E, Fartoux L, Faivre S, Blanc JF, Viret F, et al: Gemcitabine and oxaliplatin with or without cetuximab in advanced biliary-tract cancer (BINGO): A randomised, open-label, non-comparative phase 2 trial. Lancet Oncol 15: 819-828, 2014.

59. Sui M, Li Y, Wang H, Luo Y, Wan T, Wang X, Hu B, Cheng Y, Lv X, Xin X, et al: Two cases of intrahepatic cholangiocellular carcinoma with high insertion-deletion ratios that achieved a complete response following chemotherapy combined with PD-1 blockade. J Immunother Cancer 7: 125, 2019.

60. Formenti SC: Immunological aspects of local radiotherapy: Clinical relevance. Discov Med 9: 119-124, 2010.

61. Liu X, Yao J, Song L, Zhang S, Huang T and Li Y: Local and abscopal responses in advanced intrahepatic cholangiocarcinoma with low TMB, MSS, pMMR and negative PD-L1 expression following combined therapy of SBRT with PD-1 blockade. J Immunother Cancer 7: 204, 2019.

62. Jarnagin WR, Zager JS, Hezel M, Stanziale SF, Adusumilli PS, Gonen M, Ebright MI, Culliford A, Gusani NJ, Fong Y, et al Treatment of cholangiocarcinoma with oncolytic herpes simplex virus combined with external beam radiation therapy. Cancer Gene Ther 13: 326-334, 2006.

63. Churi CR, Shroff R, Wang Y, Rashid A, Kang HC, Weatherly J, Zuo M, Zinner R, Hong D, Meric-Bernstam F, et al: Mutation profiling in cholangiocarcinoma: Prognostic and therapeutic implications. PLoS One 9: e115383, 2014.

64. Mazzaferro V,El-Rayes BF, Busset MD, Cotsoglou C, Harris WP, Damjanov N, Masi G, Rimassa L, Personeni N, Braiteh F, et al: Derazantinib (ARQ 087) in advanced or inoperable FGFR2 gene fusion-positive intrahepatic cholangiocarcinoma. Br J Cancer 120: 165-171, 2019.

65. Abou-Alfa GK, Sahai V, Hollebecque A, Vaccaro G, Melisi D, Al-Rajabi R, Paulson AS, Borad MJ, Gallinson D, Murphy AG, et al: Pemigatinib for previously treated, locally advanced or metastatic cholangiocarcinoma: A multicentre, open-label, phase 2 study. Lancet Oncol 21: 671-684, 2020.

66. Suyama K and Iwase H: Lenvatinib: A promising molecular targeted agent for multiple cancers. Cancer Control 25 1073274818789361,2018

67. Chen WX, Li GX, Hu ZN, Zhu P, Zhang BX and Ding ZY: Significant response to anti-PD-1 based immunotherapy plus lenvatinib for recurrent intrahepatic cholangiocarcinoma with bone metastasis: A case report and literature review. Medicine (Baltimore) 98: e17832, 2019.
68. Klein O, Kee D, Nagrial A, Markman B, Underhill C, Michael M, Jackett L, Lum C, Behren A, Palmer J, et al: Evaluation of combination nivolumab and ipilimumab immunotherapy in patients with advanced biliary tract cancers: Subgroup analysis of a phase 2 nonrandomized clinical trial. JAMA Oncol 30: e202814, 2020.

69. Simone V, Brunetti O, Lupo L, Testini M, Maiorano E, Simone M, Longo V, Rolfo C, Peeters M, Scarpa A, et al: Targeting angiogenesis in biliary tract cancers: An open option. Int J Mol Sci 18: 418, 2017.

70. Fukumura D, Kloepper J, Amoozgar Z, Duda DG and Jain RK: Enhancing cancer immunotherapy using antiangiogenics: Opportunities and challenges. Nat Rev Clin Oncol 15: 325-340, 2018.

71. Yoshikawa D, Ojima H, Iwasaki M, Hiraoka N, Kosuge T, Kasai S, Hirohashi S and Shibata T: Clinicopathological and prognostic significance of EGFR, VEGF, and HER2 expression in cholangiocarcinoma. Br J Cancer 98: 418-425, 2008.

72. Fukumura D, Xavier R, Sugiura T, Chen Y, Park EC, Lu N, Selig M, Nielsen G, Taksir T, Jain RK and Seed B: Tumor induction of VEGF promoter activity in stromal cells. Cell 94: 715-725, 1998

73. Guion-Dusserre JF, Lorgis V, Vincent J, Bengrine L and Ghiringhelli F: FOLFIRI plus bevacizumab as a second-line therapy for metastatic intrahepatic cholangiocarcinoma. World J Gastroenterol 21: 2096-2101, 2015

74. Pan TT, Wang W, Jia WD and Xu GL: A single-center experience of sorafenib monotherapy in patients with advanced intrahepatic cholangiocarcinoma. Oncol Lett 13: 2957-2964, 2017.

75. Sun W, Patel A, Normolle D, Patel K, Ohr J, Lee JJ, Bahary N, Chu E, Streeter N and Drummond S: A phase 2 trial of regorafenib as a single agent in patients with chemotherapy-refractory, advanced, and metastatic biliary tract adenocarcinoma. Cancer 125: 902-909, 2019.

76. Schmittnaegel M, Rigamonti N, Kadioglu E, Cassará A, Rmili CW, Kiialainen A, Kienast Y, Mueller HJ, Ooi CH, Laoui D and De Palma M: Dual angiopoietin-2 and VEGFA inhibition elicits antitumor immunity that is enhanced by PD-1 checkpoint blockade. Sci Transl Med 9: eaak9670, 2017.

77. Allen E, Jabouille A, Rivera LB, Lodewijckx I, Missiaen R, Steri V, Feyen K, Tawney J, Hanahan D, Michael IP and Bergers G: Combined antiangiogenic and anti-PD-L1 therapy stimulates tumor immunity through HEV formation. Sci Trans Med 9: eaak9679, 2017.

78. Arkenau HT, Martin-Liberal J, Calvo E, Penel N, Krebs MG, HerbstRS, Walgren RA, Widau RC,MiG,Jin J,etal: Ramucirumab plus pembrolizumab in patients with previously treated advanced or metastatic biliary tract cancer: Nonrandomized, open-label, phase i trial (JVDF). Oncologist 23: e1407-e1436, 2018.

79. Marabelle A, Le DT, Ascierto PA, Di Giacomo AM, De Jesus-Acosta AD, Delord JP, Geva R, Gottfried M, Penel N, Hansen AR, et al: Efficacy of pembrolizumab in patients with noncolorectal high microsatellite instability/mismatch repair-deficient cancer: Results from the phase II KEYNOTE-158 study. J Clin Oncol 38: 1-10, 2020.

80. Ott PA, Bang YJ, Piha-Paul SA, Razak AR, Bennouna J, Soria JC, Rugo HS, Cohen RB, O'Neil BH, Mehnert JM, et al: T-Cell-Inflamed gene-expression profile, programmed death ligand 1 expression, and tumor mutational burden predict efficacy in patients treated with pembrolizumab across 20 cancers: KEYNOTE-028. J Clin Oncol 37: 318-327, 2019.

This work is licensed under a Creative Commons Attribution-NonCommercial-NoDerivatives 4.0 International (CC BY-NC-ND 4.0) License. 